\title{
Entomological and Acarological Evidence of Bloodstain Patterns in Forensic Investigation
}

\author{
Aly H. Rasmy \\ National Research Centre, Dokki, Cairo, Egypt \\ e-mail: aly_rasmy@hotmail.com
}

Blood was used as evidence in crimes since the eleventh-century when the term red-handed being used to identify criminals who had blood on their hands.

Thereafter, it was reported that insects at a crime scene transport blood shed to new locations and can alter bloodstain pattern (Parker et al., 2010). The misinterpretation of entomological alteration of bloodstain pattern may make it difficult to distinguish between guilt and innocence. The recognition of insect produced artifacts at a crime scene, and the understanding of the mechanics of insects feeding on blood and decomposing bodies are essential for proper analysis of bloodstain patterns.

The aim of the present article is to help the forensic investigators to understand the basic of bloodstain pattern analysis.

Most often, the mechanical transmission of blood from a pooled source to a blood-free surface is performed by the legs of the flies. Flies ingest blood and then regurgitate it onto a surface to allow enzymes to breakdown the blood. Later on, the flies return to suck the regurgitated blood and thus resulted in the appearance of dome shaped craters stains due to sucking process and others may show swiping due to defecation. These bloodstains could be observed nearby windows and ceiling (quoted after Benecke \& Banksdale, 2003; Benecke et al., 2000). Fly specks of fly facal spots are swipe pattern that typically exhibit a comma shape with the tail of the drop trailing to the left, right or straight of center, depending on the movement of the abdomen (quoted after Parker et al., 2010).

Roaches also produce blood droplets by their blood contaminated legs, but the droplet size is larger than that produced by flies. Also, artifact of roaches are usually recognized, as the droplet pattern reveals a series of tracks that often have a center drag mark caused by the roach dragging its under surface of abdomen tip as it walks.

However, the randomness of the stain pattern and contrasting directionally of the stain of the fecal material which looks like elongated comma shape indicate that this swipe pattern is produced by insects activity and is not a bloodstain resulting from a violent act committed during the crime.

Fleas can also be responsible for defecating partially digested blood that originated from the victim or others who were present at the scene.

This information of bloodstain evidence analysis can reveal what did and who might be involved in these actions, and what actions took place during the act of the crime. In other words, it answers the questions of who, what, where, when and how?

In this concept, the scope of forensic acarology goes further than mites as indicators of time of death or relocation of bodies or locating a suspect at the scene of a crime and may be of value in the analysis of trace cements (extracted from Perotti el al. 2009). Moreover, the anthor 2008 and 2009) reported that mites have a part to piay as indicators in case of negice or physical abuse

Here, no evidence on bloodstain pattern resulted from mite activity in the scene of a crime has been reported yet. This could be due to their tiny size and thus, bloodstains in a crime scene are easily missed and undetected by untrained eyes. At most, the blood pattern artifact caused by mites at a crime scene will not be of interest to bloodstain experts for a while, although mites are omnipresent in our domestic life. 


\section{Case study (Tallahassee, USA, 1998)}

Neighbours, who lived in the adjacent apartment of a man, reported his disappearance as well as a foul smell was grown out of his residence. When entering the apartment, bloodstain evidence was noted throughout the residence including the floor and the walls. The victim was found laying-down in the bathtub. There were also additional patterns such as pooled blood. These patterns were produced from the migratory fly larvae existing the bathtub. Additionally, empty medical bottles and a razorblade were noted close to the bathtub. The bloodstain patterns were concentrated on the bathroom door and the walls.

It was interesting that the forensic entomologist determined that these blood patterns originated from mechanical transmission and digestion of the flies present at the scene. Therefore, no violent force or impact factor created these patterns.

Thus, it was determined that this case was a suicide.

\section{REFERENCES}

Benecke, M.; Barkdale, L.; Sundermeier, J.; Reibe, S. and Ratcliffe, B.C.R. 2000. Forensic entomology in a murder case. Blood spatter artifacts caused by flies, and determination of post mortem interval by use of blowfly maggots. Zoology 103 (Suppl. 111): 106.

Benecke, M. and Barkdale, L. 2003. Distinction of bloodstain patterns from fly artifacts. Forensic Int., 137: 152-159.

Parker, M.A.; Benecke, M.; Byrde, J.H.; Hawkes, R. And Brown, R. 2010. Entomological alteration of bloodstain evidence. In Forensic entomology: The utility of arthropods in legal investigations, 539-580. Eds. J.H. Byrd and J.L. Castner, CRC Press, 580.

Perotti, M.A.; Goff, M.L.; Baker, A.S.; Turner, B.D. and Braig, H.R. 2009. Forensic acarology. Exp. Appl. Acarology, 49:3-13.

Rasmy, A.H. 2008. Mites and insects as indicators of physical abuse. Acarines, 2: 1-2.

Rasmy, A.H. 2009. Acarology and the law: History and areas for future research, 3: 1-2. 